Miscellaneous Report 169

\title{
AK GEMS SYMBOLOGY: A DESCRIPTION OF THE AK GEMS STYLE FILE
}

Patricia G. Ekberg, Michael D. Hendricks, and Jennifer E. Athey

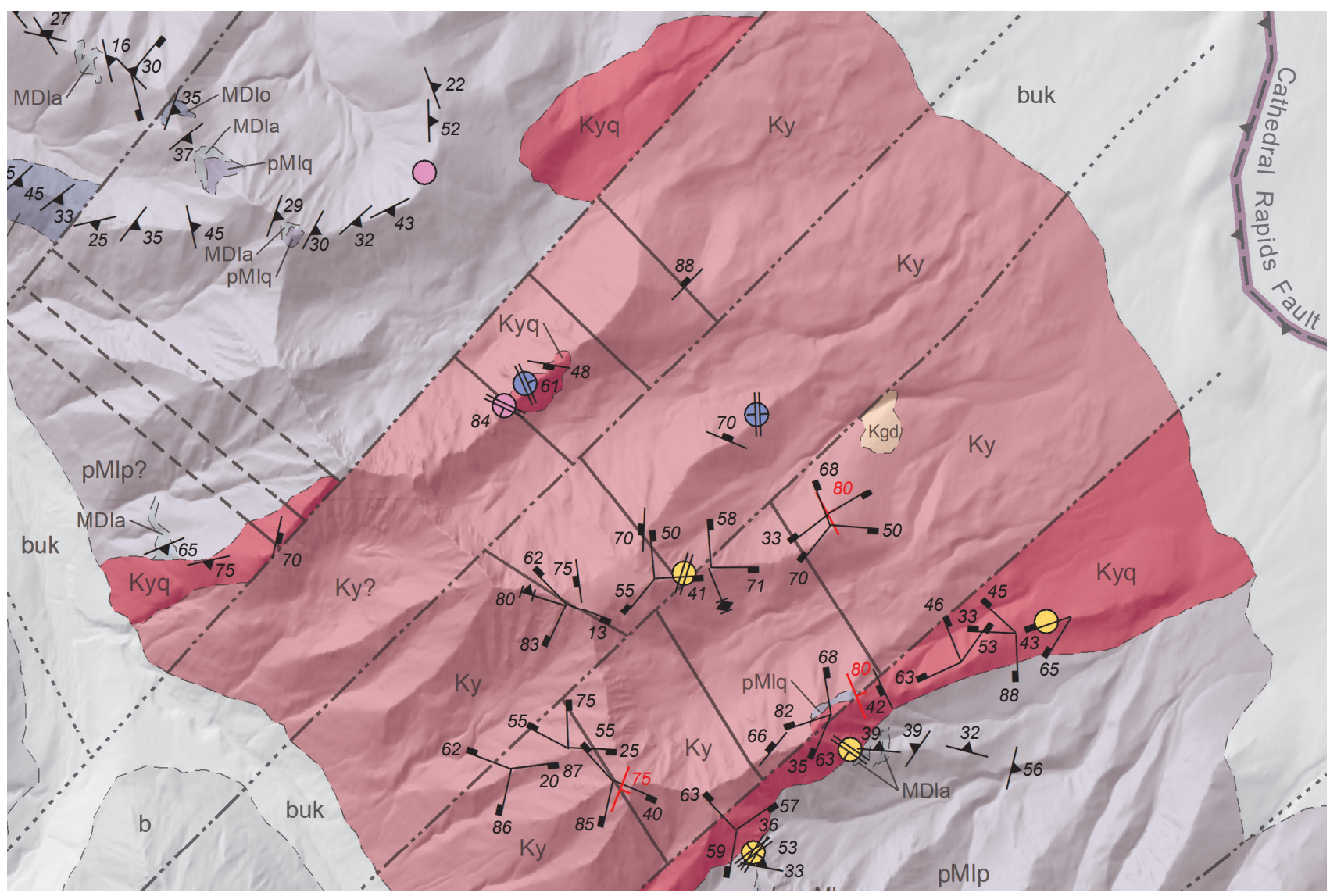

Published by

STATE OF ALASKA

DEPARTMENT OF NATURAL RESOURCES

DIVISION OF GEOLOGICAL \& GEOPHYSICAL SURVEYS 



\section{AK GEMS SYMBOLOGY: A DESCRIPTION OF THE AK GEMS STYLE FILE}

Patricia G. Ekberg, Michael D. Hendricks, and Jennifer E. Athey

Miscellaneous Report 169

State of Alaska

Department of Natural Resources

Division of Geological \& Geophysical Surveys 


\section{STATE OF ALASKA}

Mike Dunleavy, Governor

\section{DEPARTMENT OF NATURAL RESOURCES}

Corri A. Feige, Commissioner

\section{DIVISION OF GEOLOGICAL \& GEOPHYSICAL SURVEYS}

Steve Masterman, State Geologist and Director

Publications produced by the Division of Geological \&

Geophysical Surveys (DGGS) are available for free download

from the DGGS website (dggs.alaska.gov). Publications on

hard-copy or digital media can be examined or purchased in

the Fairbanks office:

Alaska Division of Geological \& Geophysical Surveys

3354 College Rd., Fairbanks, Alaska 99709-3707

Phone: (907) 451-5010 Fax (907) 451-5050

dggspubs@alaska.gov|dggs.alaska.gov

\section{DGGS publications are also available at:}

Alaska State Library,

Historical Collections \& Talking Book Center

395 Whittier Street

Juneau, Alaska 99811

Alaska Resource Library and Information Services (ARLIS)

3150 C Street, Suite 100

Anchorage, Alaska 99503

\section{Suggested citation:}

Ekberg, P.G., Hendricks, M.D., and Athey, J.E., 2021, AK GeMS symbology: A description of the AK GeMS style file: Alaska Division of Geological \& Geophysical

Surveys Miscellaneous Publication 169, 7 p. https://doi.org/10.14509/30584
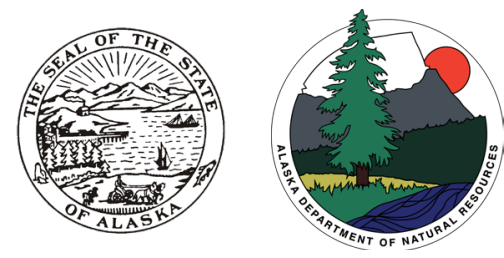


\section{Contents}

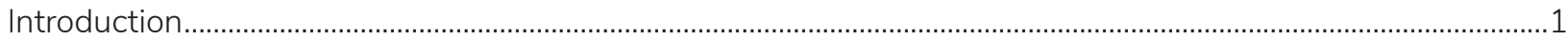

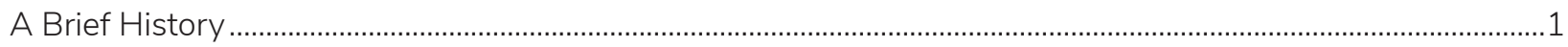

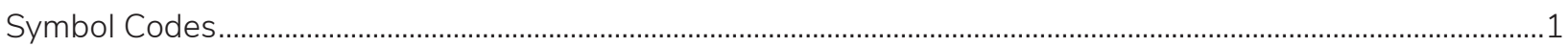

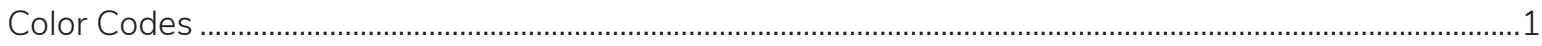

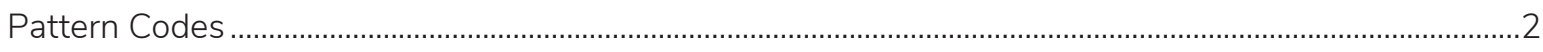

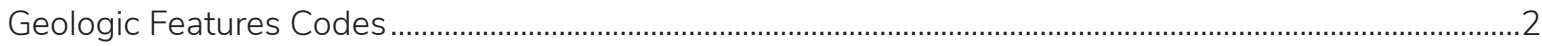

Symbol Codes for Map Unit Polygons, Map Unit Lines, and Map Unit Points ............................................2

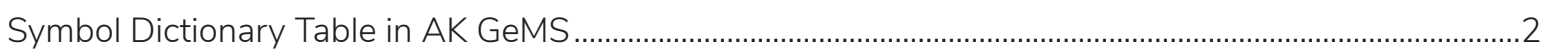

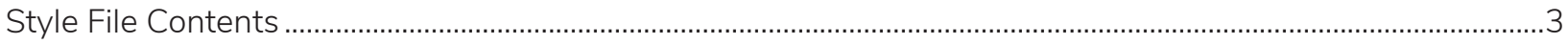

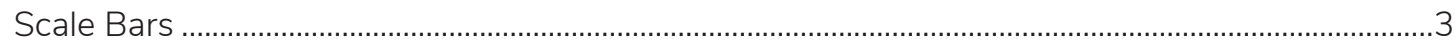

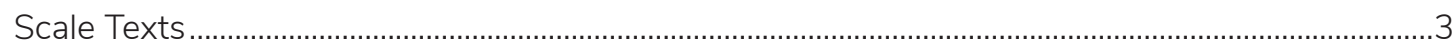

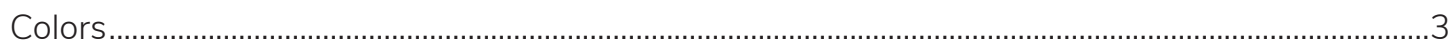

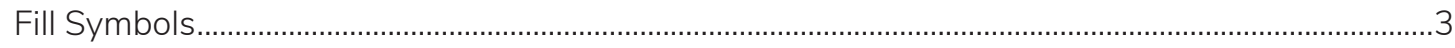

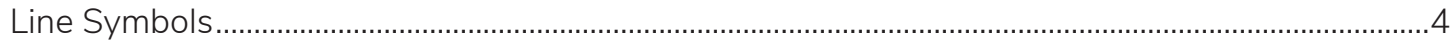

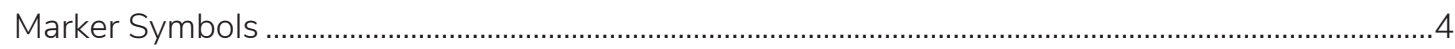

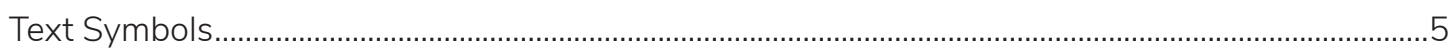

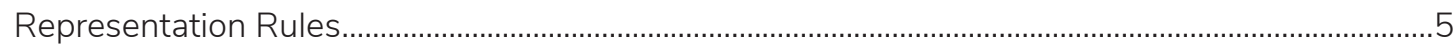

Custom Symbols and AK GeMS Symbology Documentation Excel Spreadsheet ..........................5

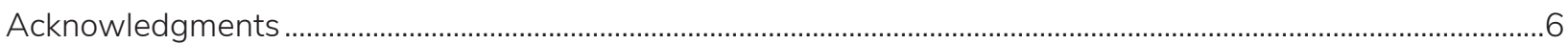

References

\section{Supplemental Files}

The following supplemental files are available to download at: https://doi.org/10.14509/30584

AK_GeMS_symology_documentation_ver_1.xlsx

AK_GeMS_symbology_ver_1.style 



\section{AK GEMS SYMBOLOGY: A DESCRIPTION OF THE AK GEMS STYLE FILE}

Patricia G. Ekberg ${ }^{1}$, Michael D. Hendricks ${ }^{1}$, and Jennifer E. Athey ${ }^{1}$

\section{INTRODUCTION}

Symbols for geologic maps are built, stored, and delivered with a database schema that is driven primarily through the use of symbol codes representing specifications found in the Federal Geographic Data Committee (FGDC) Digital Cartographic Standard for Geologic Map Symbolization publication (U.S. Geological Survey, 2006), hereafter called the FGDC standard. To represent additional visualization requirements, the Alaska Division of Geological \& Geophysical Surveys (DGGS) has created custom symbols with associated symbol codes. These code-based symbol specifications are stored digitally and available to ESRI GIS software in a style file, as specified in the U.S. Geological Survey (USGS) Geologic Map Schema (GeMS), a standardized database schema (database design) for the publication of geologic maps (U.S. Geological Survey National Cooperative Geologic Mapping Program, 2020).

This document describes the organization and content of the current style file used by DGGS for the Alaska GeMS map production standard. The Alaska GeMS map production standard (hereafter referred to as AK GeMS) is a standard schema based on the USGS GeMS schema that has been modified and expanded to better suit DGGS map and data requirements, including functionality for a multi-map database version of the schema. Database tables and field names in AK GeMS are similar to those in USGS GeMS, but do not match exactly. For example, AK GeMS uses underscores in field and table names (such as contacts_and_faults) to facilitate storage in a PostgreSQL database, while USGS GeMS uses pascal case field and table names (such as ContactsAndFaults). All accompanying files are available to download from the DGGS website: https://doi.org/10.14509/30584.

\section{A BRIEF HISTORY}

The DGGS GeMS Symbology style file is derived from a style file built by the Geological Survey of Canada (GSC), who painstakingly created a majority (but not all) of the symbols in the FGDC standard. From 2014 until 2019, DGGS built upon this foundation and added a limited number of custom symbols in a set of style files named DGGS_Map_Symbolization.style, DGGS_Map_Symbolization_ver2.style, and the short-lived DGGS_Map_Symbolization_ver3.style.

In 2020, DGGS initiated a significant update to the geologic map style file that includes a change in how custom symbol codes are codified and used, and included the following elements: colors, map_ unit_point marker symbols, and map_unit_lines line symbols based on the standard FGDC color codes. In addition, the name of the style file was updated to align with AK GeMS and, as of fall 2020, is on the first version.

\section{SYMBOL CODES}

The FGDC standard identifies symbol codes for colors, patterns, and geologic features (USGS, 2006). Below is a short description of each and how the AK GeMS style employs these codes.

\section{Color Codes}

The FGDC standard specifies a four-digit color Cyan-Magenta-Yellow-Black (CMYK) code to represent colors. These CMYK colors were designed to closely match the USGS original offset-printed, process-ink

${ }^{1}$ Alaska Division of Geological \& Geophysical Surveys, 3354 College Rd., Fairbanks, Alaska 99709-3707. 
color chart, "Printing Colors and Screens in Use by the U.S. Geological Survey for Geologic and Hydrologic Maps" [yellow/magenta/cyan version], which has long been used at the USGS for choosing colors on geologic maps. The FGDC color chart contains the same colors as the original offset-printed USGS color chart; however, the old color codes indicating the YMC (yellow/magenta/cyan) values have been updated to show CMYK (cyan/magenta/yellow, with black = 0) values, to conform to industry standards.

For each code, each digit represents the percent of cyan $(\mathrm{C})$, magenta $(\mathrm{M})$, yellow $(\mathrm{Y})$, or black $(\mathrm{K})$. Color codes use the following abbreviations: $A=8$ percent; $1=13$ percent; $2=20$ percent; $3=30$ percent; $4=40$ percent; $5=50$ percent; $6=60$ percent; $7=70$ percent; X=100 percent. For example, the code $40 \mathrm{X} 0$ represents a yellow-green color that is comprised of 40 percent cyan, 0 percent magenta, 100 percent yellow, and 0 percent black.

While DGGS employs the four-digit CMYK-based code, the FGDC color chart also provides a three-digit generic lookup-table number that can be used to reference a color. A lookup table and scripts are available from DGGS to assist in converting between various color codes and models.

\section{Pattern Codes}

FGDC standard patterns are identified by a pattern number, which includes a 3-digit number followed by a letter identifying the color of the pattern. For example, code 101-K represents pattern 101 (a pattern comprised of randomly dispersed small open ovals and dots) shown in black (K). Pattern 101 can also be shown in cyan (code 101-C), magenta (code 101-M), or dropout (code 101-DO). Standard patterns are in section 38 of the FGDC standard and on the accompanying pattern chart. The FGDC pattern chart also provides a three-digit lookup-table symbol number that can be used to reference a pattern, however, DGGS employs the number and letter-based coding.

\section{Geologic Features Codes}

The FGDC standard employs a dot-based hierarchical numbering logic for its symbol codes. For example, symbol code 1.1.1. is "Contact-Identity and existence certain, location accurate." The first digit indicates that it is within the Contact, Key Beds, and Dikes category, the second digit indicates it is within the Contacts sub-category, and the third digit is the specific symbol. Some symbols, however, do not have a sub-category; for example, code 6.1 is "Horizontal bedding."

For convenience, DGGS pads single-digit FGDC symbol code values with a leading zero to assist in sorting. For example, FGDC code 1.1.1 becomes 01.01.01. The symbol code associated with each feature in a GeMS geodatabase is stored in the feature classes' symbol field (type string, length 10).

\section{Symbol Codes for Map Unit Polygons, Map Unit Lines, and Map Unit Points}

Map unit polygons, lines, and points in AK GeMS use the four-digit color CMYK-based color code described earlier in the color codes section.

\section{Symbol Dictionary Table in AK GeMS}

A symbol lookup table, named symbol_dict in AK GeMS, provides access to the symbol code's description, derived when available from the FGDC standard. For example, symbol code 02.03.01 has a description of "Low-angle fault (unknown or unspecified sense of slip)_-Identity and existence certain, location accurate." 


\section{STYLE FILE CONTENTS}

All the FGDC symbols that were originally in the GSC style file are still included in this DGGS style file; even those that are not currently used in AK GeMS (fig. 1).

A list of the folders in the style file, and the changes or additions (if any) DGGS has made, is provided below:

\section{Scale Bars}

The Scale Bars folder contains one scale bar style representative of the specifications found in the FGDC standard section 35 for scale bars and scale text.

\section{Scale Texts}

The Scale Texts folder contains one scale text style representative of the specifications found in the FGDC standard section 35 for scale bars and scale text.

\section{Colors}

The Colors folder contains all colors from the color chart of the FGDC standard. Color symbols allow for colors to be viewed and accessed in the ArcMap color selector palette. Although this may be helpful with some small color sets, there are limited number of color patches that can be viewed in the ArcMap selector palette, and there are too many FGDC standard colors to make this feature currently usable.

\section{Fill Symbols}

The Fill Symbols folder contains many fill symbols that can be categorized into the groups listed below. The symbol code identifying each fill symbol differs according to these groups.

\section{Map Unit Fill Symbol Based on USGS Suggested Colors}

Colors and patterns can be used to fill map units and other polygons in polygon feature classes in AK GeMS (map_unit_polys, overlay_polys, etc.). The colors from the USGS Suggested Colors for Geologic Maps* plate (USGS, 2006) are included as fill symbols in the style file. The fill symbols are coded by the four-digit CMYK color code (e.g., 40X0). This is particularly useful when using the option to symbolize features according to a style file based on values in a field (which you can now do in ArcGIS Pro). In AK GeMS, the four-digit color code (e.g., 40X0) is used as the symbol code in the symbol field for map unit polygons.

\section{FGDC Standard Fill Symbols}

Other FGDC standard fill symbols are identified by their dot-based FGDC symbol codesimilar to the convention used for line and points symbols. These FGDC standard fill symbols are either based on a single color, such as symbol code 01.02 .42 (area of outcrop), or built on a standard

\footnotetext{
*The color and pattern suggestions in this publication are examples of what could be used on a geologic map and do not necessarily form a strict or complete standard. Many factors should be considered when selecting colors for a geologic map. Additional guidance and recommendations are provided in section 5, "Guidelines for Map Color and Pattern Selection", of the introductory text in the FGDC standard (U.S. Geological Survey, 2005.).
}

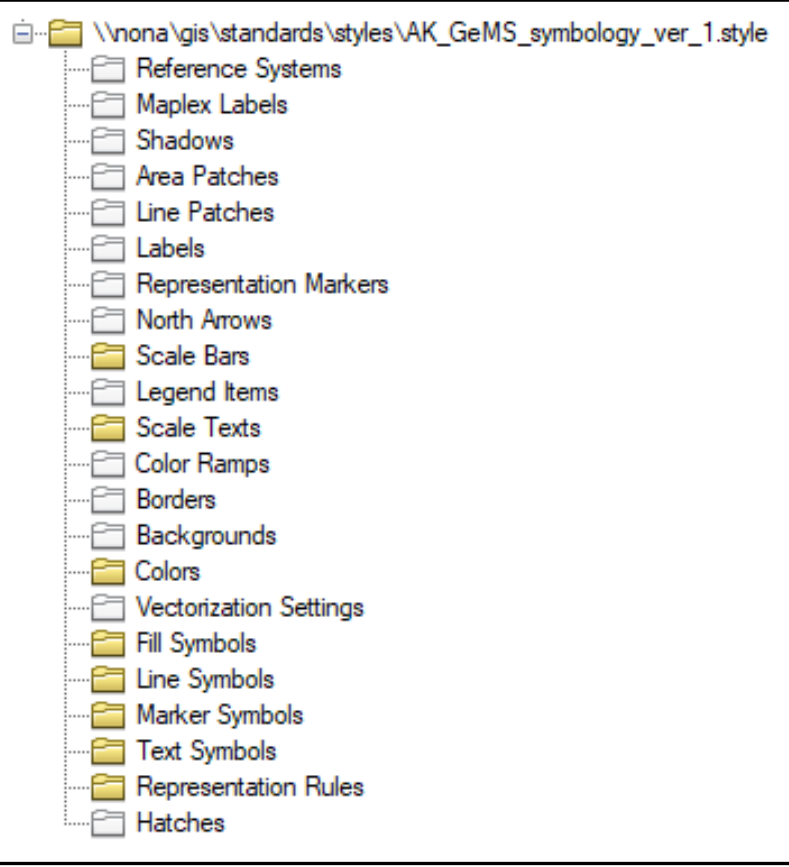

Figure 1. The contents of AK_GeMS_symbology_ver_1.style when viewed in the ArcMap Style Manager window. Yellow folders contain symbols; grey folders are empty. 
pattern, such as 20.07 (hazardous waste site pattern 226-R with line weight of $0.25 \mathrm{~mm}$ outline). There are currently 17 FGDC standard fill symbols from various FGDC standard sections.

\section{FGDC Patterns as Picture Fill Symbols}

To make the patterns as picture fill symbols, pattern swatch images were downloaded as .png files from the full FGDC repository available on GitHub (Quinn, 2017), loaded into the ArcMap symbol selector, and scaled to draw at the correct size. Currently, a limited number of patterns are available, but more patterns will be added in future versions.

\section{Line Symbols}

The Lines Symbols folder contains many line symbols that are used in different line feature classes in AK GeMS (contacts_and_faults, structure_lines, map_unit_lines, etc.). Line symbols can be categorized into the groups listed below. The symbol code identifying each line symbol differs according to these groups.

\section{AK GeMS Map Unit Line Symbols Based on USGS Suggested Colors}

These custom line symbols are used to symbolize features in the map_unit_lines feature class in AK GeMS. The symbol code for each map unit's line symbol is the FGDC color code that corresponds to the color of a map unit (e.g., 40X0). DGGS is creating these map unit line symbols as they are needed, so there is not a line for every FGDC suggested color.

\section{FGDC Standard Line Symbols}

Almost all standard FGDC line symbols (created by the GSC) are in this folder. These symbols are most often used in the contacts_and_faults and structure_lines feature classes.

\section{AK GeMS Custom Line Symbols}

DGGS has started to create custom line symbols to be used when there is no FGDC symbol available for a geologic feature, or if an FGDC symbol needs to be repurposed. These custom symbols are explained further in the next section.

\section{Marker Symbols}

The Marker Symbols folder contains many marker symbols that are used in different point feature classes in AK GeMS (e.g., orientation_points, geochron_points, map_unit_points, etc.). Marker symbols can be categorized into the groups listed below. The symbol code identifying each marker symbol differs according to these groups.

\section{AK GeMS Map Unit Point Symbols}

These custom point symbols are used to symbolize features in the map_unit_points feature class in AK GeMS. The symbol code for each map unit point symbol is the FGDC color code that corresponds to the color of a map unit (e.g., 40X0). There is a map unit point marker symbol for every FGDC suggested color.

\section{FGDC Standard Marker Symbols}

Almost all standard FGDC marker symbols (created by the GSC) are in this folder. This includes marker symbols for point features (e.g., symbol 06.02 represents a bedding symbol in the orientation_points feature class) and marker decorations that can be placed at a point or along a line (e.g., symbol 02.11.01 represents a ball and bar decoration to show normal offset of a fault). 


\section{Text Symbols}

DGGS does not use text symbols directly for symbolization based on a field in an attribute table. Symbol text specifications can be used as a starting point and then modified to create labels or annotation feature classes.

\section{Representation Rules}

DGGS converted many FGDC symbols and patterns into cartographic representation rules, however, following the release of ArcGIS Pro, ESRI is moving away from cartographic representations. As a result, current and future DGGS products do not include cartographic representations. Representation rules remain in this style file in case they are helpful to other organizations and are organized by FGDC symbol or pattern code.

\section{Custom Symbols and AK GeMS Symbology Documentation Excel Spreadsheet}

The AK_GeMS_symbology_documentation_ver_1 spreadsheet was developed to explain the custom symbols created by DGGS for use in AK GeMS. Symbols are grouped into seven appendices, each with a separate worksheet tab, depending on their origin and use in AK GeMS. Due to Microsoft Excel characterlength limitations for worksheet names, some abbreviations are used in tab/appendices naming.

Each tab has a Symbol Type column that explains a symbol's general use and identifies whether the symbol is an FGDC Standard or DGGS custom symbol (table 1).

Table 1. Definitions of the symbol type definitions in the AK_GeMS_symbology_documentation_ver_1 spreadsheet.

\section{Symbol Type}

FGDC Primary

FGDC Secondary

FGDC Decoration Secondary

FGDC Alternate
FGDC Decoration

\section{Definition}

The primary and expected FGDC symbol used to draw a feature type stored in an AK GeMS database.

A substitute FGDC symbol used to draw a feature type stored in an AK GeMS database. These symbols should not be used without coordination with the AK GeMS admin staff. See the assoc_ symbol_code field for this secondary symbol's primary symbol.

A FGDC symbol used to enhance or decorate a symbol. For example, a plunge direction.

A substitute FGDC symbol used to enhance or decorate a symbol. For example, a plunge direction. These symbols should not be used without coordination with the AK GeMS admin staff. See the assoc symbol_code field for this secondary symbol's primary symbol.

An alternate FGDC symbol used to draw a feature with special characteristics stored in an AK GeMS database. A typical example of an alternate symbols is an orientation point with multiple observations at one locality. See the assoc_symbol_code field for this symbol's primary symbol.

FGDC Not Used by AK GeMS The FGDC symbol is not currently used by DGGS.

FGDC Not Available in Style The FGDC symbol is not available in the current style file.

Custom symbol made by DGGS staff for features that do not have AK GeMS Custom a standard FGDC symbol or in cases where a FGDC symbol needs to be repurposed. 
Appendices A-G (viewable as separate tabs in the supplemental excel file) describe and explain variations of the custom symbols in the FGDC and AK GeMS standards. In each tab, information includes the AK GeMS category and type; and a feature description that provides details about the symbolized feature that might not be in the feature attribute table. The appendices are as follows:

FGDC Standard Symbols - This tab explains all the FGDC standard symbols in the style file as they are found in the FGDC standard. Symbols are listed by their FGDC symbol code, with padded zeros (e.g., 01.01.01).

Compound FGDC Symbols - This tab explains compound symbols that are created from two or more standard FGDC symbols. Typically, these are lines that need to have a repeating decoration along them, or a second symbol level of line color. Symbols are listed by the first FGDC symbol code / second symbol code (e.g., 02.04.03/02.11.04).

Custom Symbs w FGDC Sections - This tab explains custom symbols that fit into the established FGDC standard sections. The symbol codes all start with the FGDC section and subsection into which the feature corresponds, followed by 'ak' and a unique number (e.g., 01.03.ak.01).

Custom Symbs NO FGDC Sections - This tab explains custom symbols that DO NOT fit into the established FGDC standard sections. The symbol codes all start with 'ak', followed by a group number (starting at 101 and assigned based on AK GeMS category), and followed by a unique number (e.g., ak.101.01).

Symbols with alternate colors - This tab explains FGDC and AK GeMS custom symbols that need to be shown in alternate colors. In the FGDC standard, the notes on usage for many symbols indicate that a symbol "may be shown in other colors". It can be hard to show the same symbol in different colors on the same map using dynamic symbolization. The default color, as shown in the FGDC standard, is the default color for the symbol in the style file. When an alternate color is needed, a custom symbol is created that uses the standard symbol code and the CMYK color code of the alternate color. For example, FGDC standard symbol 18.56 is a volcanic vent that has a default red color. When needed to be shown in black, the feature is symbolized with code 18.56.XXX0. To conform with the FGDC color convention of $\mathrm{K}=0$, the code XXX0 is used to represent black.

map_unit_point Symbols_-This tab explains the convention for symbolizing map unit points. The symbol code for map unit points is the FGDC color code that corresponds to each map unit's color.

map_unit_line Symbols_-This tab explains the convention for symbolizing map unit lines. The symbol code for map unit lines is the FGDC color code that corresponds to each map units color.

\section{ACKNOWLEDGMENTS}

The authors thank reviewer Taryn Lindquist for her thoughtful comments. This work was funded by Cooperative Agreement G19AC00411 with the USGS National Geologic Map Database and State of Alaska General Funds. 


\section{REFERENCES}

U.S. Geological Survey, 2005, Selection of colors and patterns for geologic maps of the U.S. Geological Survey: U.S. Geological Survey Techniques and Methods 11-B1, 12 p., 1 sheet. https://pubs.usgs.gov/ $\mathrm{tm} / 2005 / 11 \mathrm{~B} 01 /$

2006, FGDC Digital Cartographic Standard for Geologic Map Symbolization (PostScript Implementation): U.S. Geological Survey Techniques and Methods 11-A2. http://pubs.usgs.gov/ $\mathrm{tm} / 2006 / 11 \mathrm{~A} 02 /$

U.S. Geological Survey National Cooperative Geologic Mapping Program, 2020, GeMS (Geologic Map Schema)—A standard format for the digital publication of geologic maps: U.S. Geological Survey Techniques and Methods, book 11, chap. B10, 74 p. https://doi.org//10.3133/tm11B10

Quinn, Daven, 2017, FGDC Geologic Patterns for the Web: Github [webpage], last accessed 10/02/2020. https://github.com/davenquinn/geologic-patterns 